\title{
Defining the Learning Curve is Necessary to Improve Oocyte Retrieval Skills: Lessons from A Single High- Volume Reproductive CenterDefining the Learning Curve is Necessary to Improve Oocyte Retrieval Skills: Lessons from A Single High-Volume Reproductive Center
}

\section{Mingzhu Cao}

Third Affiliated Hospital of Guangzhou Medical College

\section{Danming You}

Southern Medical University Nanfang Hospital

\section{Sichen Li}

Third Affiliated Hospital of Guangzhou Medical College

Yixuan Wu

Third Affiliated Hospital of Guangzhou Medical College

\section{Haiying Liu}

Third Affiliated Hospital of Guangzhou Medical College

\section{Qing Huang}

Third Affiliated Hospital of Guangzhou Medical College

Jianqiao Liu ( $D$ ljq88gz@163.com )

Third Affiliated Hospital of Guangzhou Medical College

\section{Research}

Keywords: Learning curve, oocyte retrieval, training

Posted Date: July 29th, 2020

DOI: https://doi.org/10.21203/rs.3.rs-44933/v1

License: (a) (1) This work is licensed under a Creative Commons Attribution 4.0 International License. Read Full License 


\section{Abstract}

Objective: The objective of this study was to determine the novice trainee's learning curve for oocytes retrieval procedure through assessment of oocytes retrieval efficiency, operative time and other operative characteristics.

Methods: This retrospective cohort study included 200 consecutive patients undergoing transvaginal ultrasound guided oocytes retrieval procedure. Those patients underwent oocyte retrieval procedure by a single operator and one experienced supervisor. Their clinical data, including demographic data, ovarian stimulation cycle information, surgical procedure, and laboratory data were collected over 3 months. CUSUM analyses based on the operative time were performed to determine the learning curve.

Results: The mean operative time was $10.10 \mathrm{~min}$. Based on the CUSUM plot of operative time, the learning curve can be divided into three separated phases, phase 1 (case 1 to case 49) was learning phase, phase 2 (case 50 to case 130) was acquisition phase, and phase 3 (case 131 to case 200) was proficiency phase. The operative time was significantly shortened from phase 1 to phase 3 (phase 1, $13.37 \pm 4.83 \mathrm{~min}$; phase 2, $10.21 \pm 3.30 \mathrm{~min}$; phase $3,7.67 \pm 3.24 \mathrm{~min}, \mathrm{P}<0.001)$. The oocyte retrieval efficiency was also notably improved from phase 1 to phase 3 ( $78.2 \%$ to $100 \%$ based on method 1 to determine oocyte retrieval efficiency, and $104.2 \%$ to $121.1 \%$ based on method 2 to determine oocyte retrieval efficiency). The retrieved oocytes number, the fertilization rate, clinical pregnancy rate among the three phases showed no significant differences. No patients had severe adverse events. As determined by multiple linear regression, learning phase is the only independent predictor of oocyte retrieval efficiency.

Conclusion: Trainees practice transvaginal ultrasound guided oocytes retrieval are expected to achieve a stabilized procedure over consecutive training cases, with acquisition of the skills at 49 cases, and proficiency at 130 cases. Cumulative operative experience can improve the operative time and oocytes retrieval efficiency, but showed minimal influence on retrieved oocytes number and reproductive outcomes.

\section{Introduction}

In 2016, over 500,000 oocytes retrieval cycles were performed in China for infertile couples every year [1]. And the number of oocyte retrieval cycles is increasing year by year [1]. Oocytes retrieval is one critical procedure of ART cycles. It is commonly considered as an easily-learned and minimally-invasive operation. However, the procedure still remains to be technically challenging, which requires skills in sonography, reproductive medicine, and minimal invasive surgeries. With such large number of ART cycles performed in China, a lack of formal and standard training program of ART procedures is one common problem.

Defining the learning curve for the individual trainee is one necessary step of training procedure and quality control. For novice trainee, performing the procedure under the supervision of an experienced operator is a common practice. However, a tailored learning curve of the individual trainee has not been widely used. Thanks to Cumulative summation test (CUSUM) approach, a learning curve with distinct learning phases can be precisely evaluated. CUSUM plot has already been demonstrated to be effective to assess the proficiency level of a trainee by plotting the incremental changes in the measured outcomes during the learning process [2].

To the best of our knowledge, no required practice number of oocytes retrieval is established for Chinese novice trainees in ART field. And, there is no such study systematically examined the learning curve of oocytes retrieval 
procedures in Chinese reproductive institutions. The hypothesis of this study was that the cumulative experience from high-volume cases can help to improve the oocyte retrieval operative skills. The purpose of this study was to define the learning curve of transvaginal ultrasound guided oocytes retrieval of a single trainee, and provide helpful references for novice operators during their training process.

\section{Materials And Methods}

\subsection{Hospital settings and patients inclusion}

This is a retrospective cohort study conducted at the locally largest Reproductive Center. Consecutive patients requiring transvaginal ultrasound guided oocytes retrieval by a single operator were recruited from October to December 2019. The operator had 3-year experience in sonography and reproductive medicine, but no previous experience in oocytes retrieval procedure. Every patient signed informed consent to the collection of their clinical data for research use. The study protocol was approved by the hospital ethics committee (No. 2020-037).

Data from patients met the following criteria were excluded. 1) Poor responders were excluded based on Bologna criteria (Women who followed at least 2 of the following 3 criteria. Women aged $\geq 40$ years old, women with previous poor response with no more than 3 oocytes retrieved using a conventional stimulation protocol, and women with abnormal ovarian reserve test with antral follicle number $(\mathrm{AFC})<7$ follicles and anti-Mullerian hormone $(\mathrm{AMH})<1.1 \mathrm{ng} / \mathrm{ml}$ [3]. 2) Hyper-responders, determined as women with $\geq 15$ oocytes to be retrieved [4].

\subsection{Procedure technique}

The demographic data of all patients including female age, male age, infertile duration, infertile type and factors, body mass index (BMI), antral follicle counting (AFC), anti-Mullerian hormone (AMH), were collected.

Standard ovarian stimulation protocols were provided based on women's age, ovarian reservation function, ovarian response in the previous cycles, patients' and physicians' preferences. Majority of women received ovarian stimulation using gonadotropin releasing hormone $(\mathrm{GnRH})$ agonist and antagonist protocols. Otherwise, microflare or luteal phase stimulation were provided. Gonadotropin (Gn) were administrated using folliclestimulating hormone (FSH, either Gonal-F, Merck Serono, Modugno, Italy, or Puregon, MSD Organon, Oss, Netherlands), human menopausal gonadotropin (HMG, Livzon, Zhuhai, China), or luteinizing hormone (LH, Luveris, Merck Serono, Modugno, Italy). Follicle growth was monitored regularly using transvaginal ultrasound. When at least two follicles reached an average diameter at $18 \mathrm{~mm}$, or at least three follicles reached an average diameter at $17 \mathrm{~mm}$, human chorionic gonadotropin (HCG, Merck Serono, Geneva, Switzerland, or Livzon, Zhuhai, China) were used for triggering of ovulation.

The single trainee performed oocyte retrieval under the monitoring of a 7-year experienced tutor. The tutor was responsible for the supervision of the trainee during the entire learning period, until the trainee had the final assessment of technique proficiency examination after performing about 300 cases of oocyte retrieval.

Oocytes were aspirated about 36- to 37-hour following HCG triggering. The whole procedures were conducted under general anesthesia (Propofol, Libang Pharmaceutical, Xi'an, China) or with the help of pain relief drug (Ibuprofen, SmithKline \& French Laboratories Ltd., Tianjin, China). The aspirations were conducted using an 18gauge needle under vacuum and guided by transvaginal ultrasound monitoring. The vacuum suction pressure was set to about 150-160 $\mathrm{mmHg}$, and kept constant pressure during the procedures. Follicles with estimated 
size $\geq 10 \mathrm{~mm}$ were aspirated. The aspirants were transferred to the embryologist immediately, and the recovered oocytes were counted and further inseminated with sperms through either in vitro fertilization (IVF) or intracytoplasmic sperm injection (ICSI) based on the partners' sperm quality.

At the end of oocytes retrieval, a vaginal checking with gauze was performed to prevent or stop the oozing at puncture sites. If the vaginal bleeding cannot be stop within around one minute. One or two gauze were packed inside vaginal for vaginal compression to stop the bleeding. Patients with vaginal compression to stop prolonged vaginal bleeding at puncture sites were also recorded and marked as "vaginal bleeding" in this study.

Up to 2 embryos were transferred at either cleavage stage or blastocyst stage based on the number and quality of embryos and the age of female patients. The remaining usable embryos were cryopreserved by vitrification.

\subsection{Study outcomes}

The primary outcome measured of the study was operative time and oocytes retrieval efficiency. The operative time was used for determining the learning curve, and defined as the time duration between the operator started the first puncture and operator finish the vaginal checking. The oocytes retrived efficiencies were measured by dividing the number of oocytes retrived by the number of oocytes expected as determined by transvaginal ultrasound on trigger day, and were calculated based on the following methods. Method 1: Oocytes retrived efficiency $(\%, \geq 10 \mathrm{~mm})=$ number of oocytes retrived/number of follicles sized $\geq 10 \mathrm{~mm}$ on trigger day * $100 \%$. Method 2: Oocytes retrived efficiency $(\%, \geq 14 \mathrm{~mm})=$ number of oocytes retrived/number of follicles sized $\geq$ $14 \mathrm{~mm}$ on trigger day * $100 \%$.

The secondary outcomes assessed here including number of oocytes retrieved, fertilization rate, number of usable embryos on day 3, and number of embryos with top quality. The fertilization rate was evaluated as the proportion of 2 pronucleus oocytes out of number of oocytes inseminated or MII injected based on either IVF or ICSI performed. Embryo quality was evaluated on day 3 based on the assessment of blastomere number, extent of fragmentation and the symmetry of blastomere. The total score of each embryo were calculated by the sum of above parameters' evaluation as reported [5]. Usable embryos on day 3 were those with $>5$ blastomere, $<50 \%$ fragmentation and an overall score $>5$ [5]. Quality of blastocysts was evaluated based on the Gardner grade [6]. Embryos with top quality were evaluated on day 3 , and those with 8 to 10 regularly formed blastomere, no more than $20 \%$ fragmentation were marked as top quality.

Reproductive outcomes including number and reasons of cancelled embryo transfer cycles, stage and number of embryos transferred, clinical pregnancy rate, biochemical pregnancy rate, miscarriage rate, ectopic pregnancy rate, ongoing pregnancy rate were also measured. Clinical pregnancy rate was calculated as the proportion of patients with clinical pregnancy, who showed gestational sac on gestation 5 to 6 weeks, out of patients with embryos transferred. Miscarriage rate was calculated as the proportion of patients with spontaneous miscarriage out of patients with clinical pregnancy. Biochemical pregnancy rate was defined as the proportion of patients with biochemical pregnancy, who showed positive serum HCG levels but no gestational sac under sonography examination, out of the patients with embryo transfer. Ectopic pregnancy rate was defined as the proportion of patients with ectopic pregnancy whose gestational sac reside out of intrauterine cavity, out of those with embryo transfer. Ongoing pregnancy rate was defined as the proportion of patients with the presence of viable intrauterine fetus at gestational 14 weeks as confirmed by ultrasound out of those with embryo transfer. 
The safety outcome was the number of vaginal bleeding at puncture site. No other complications, for instance, intraperitoneal bleeding, infection, organ injuries, occurred during the study period.

\subsection{Statistical analysis}

All statistical analyses were processed using SPSS 19.0 (Chicago, Illinois, US). Continuous data are demonstrated as mean \pm standard deviation (SD) if they followed a normal distribution, and median and interquartile range (IQR) if not. Categorical data are demonstrated as numbers and proportions. Statistical analyses were performed using one way ANOVA for normally distributed continuous data. Nonparametric Kruskal-Wallis tests were used for data not following normal distribution. Chi-square tests and Fisher's exact test were used to compare the categorical data. A $P$ value $<0.05$ was considered as statistically significant.

A CUSUM method was performed to determine the changing trend of operative time across the case series. The mean operative time was selected as a reference value. The sequential differences between the mean operative time and each individual operative time $\left(\mathrm{D}_{\mathrm{OT}}\right)$ were calculated. Then the CUSUM for each case was calculated as summation of $\mathrm{D}_{\mathrm{OT}}$ of this case and the previous sum of operative time differences. These summations of case series yield a curve showing the trend of change in operative time, and help to determine the critical points when a change of learning phase occurs. Therefore, the learning curve based on the operative time was demonstrated by plotting the CUSUM outcomes. Multiple linear regression analyses were performed to determine the predictor of oocytes retrieval efficiencies. A stepwise regression approach was used to eliminate the inter-relationship among the independent variables. Possible confounders (phases of learning curve, female age, AFC, AMH, total Gn days for ovarian stimulation, total Gn doses for ovarian stimulation, endometrial thickness on trigger day, operative time, etc) which might affect the oocytes retrieval efficiencies were introduced into the regression analyses.

\section{Results}

A total of 200 consecutive patients operated by a single operator were included. The mean operative time was $10.1 \pm 4.29 \mathrm{~min}$. $\mathrm{D}_{\mathrm{OT}}$ were calculated by deduction of each individual operative time and the mean operative time. The sum of those $D_{\text {OT }}$ based on the case sequence were plotted to form a CUSUM (Fig. 1). CUSUM analysis of operative time shows 3 distinguished phases (Fig. 1A). Within phase 1 (case 1 to case 49, Fig. 1B), the learning phase, an increase trend in the summation was observed until the sum reaches a peak at case 49. During phase 2 (case 50 to case 130, Fig. 1C), the acquisition phase, the summation plateaued with minor fluctuation. Phase 3 (case 131 to case 200, Fig. 1D), the proficiency phase began with an inflexion at case 131, and then sum gradually reduced to the baseline. The first vertical line in Fig. 1A represents the transition from Phase 1 (learning phase) to Phase 2 (acquisition phase) of learning curve, and the second vertical line in Fig. 1A represents the transition from Phase 2 (acquisition phase) to Phase 3 (proficiency phase) of the learning curve for oocytes retrieval.

Table 1 summarizes the demographic information of patients from all 3 phases of learning curve who underwent oocytes retrieval. The baseline clinical characteristics among the three consecutive phases showed no significant differences, except for $\mathrm{AMH}$, which was obviously lower in phase 3 (median $2.11 \mathrm{ng} / \mathrm{ml}$ ) than those from phase 1 (median $2.73 \mathrm{ng} / \mathrm{ml}$ ) and 2 (median $2.92 \mathrm{ng} / \mathrm{ml})(P=0.036)$. 
Table 1

Comparisons of demographic characteristics

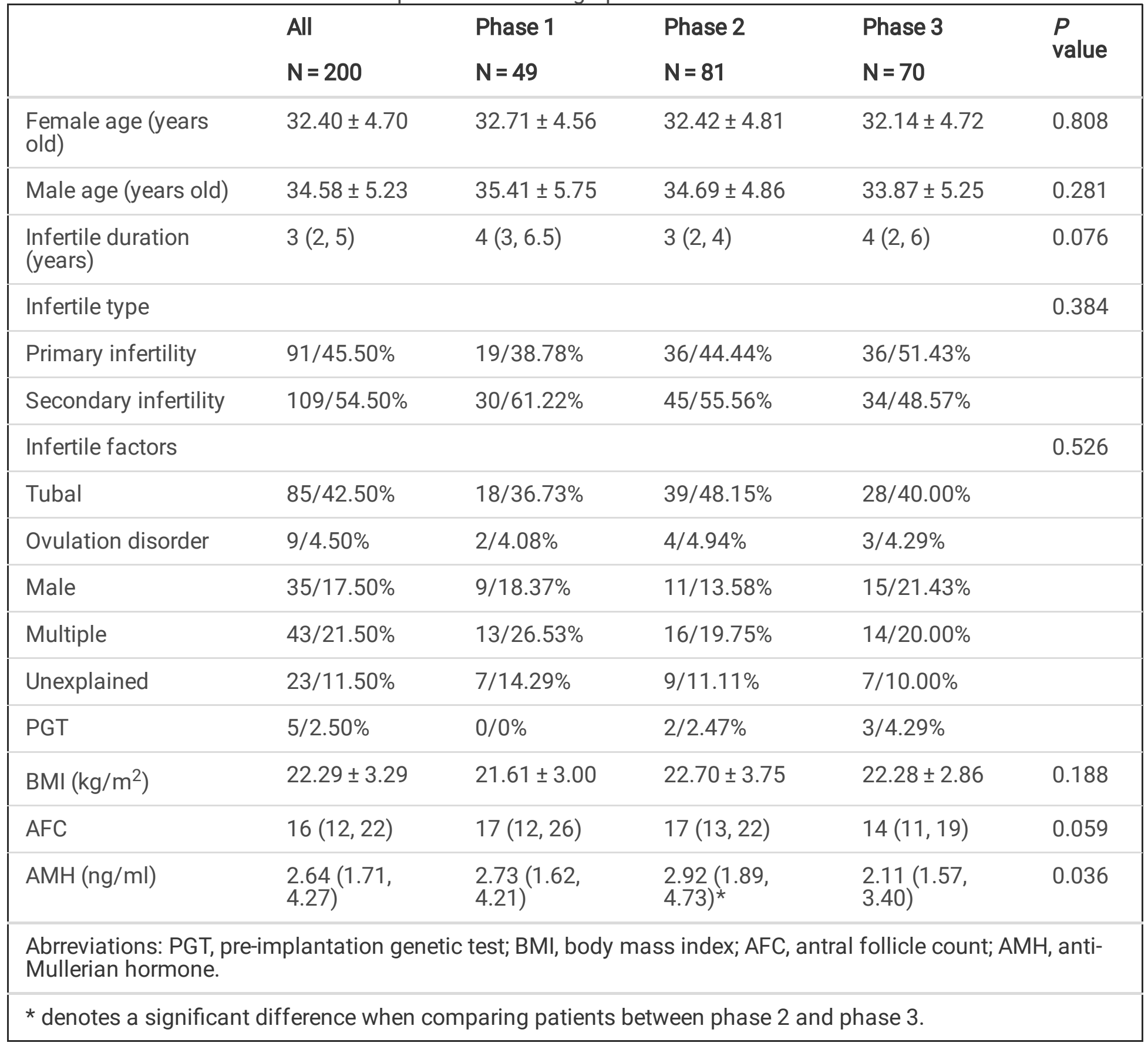

Table 2 shows the comparisons of ovarian stimulation cycle related characteristics of patients from all 3 phases of learning curve. The current ovarian stimulation cycle, ovarian stimulation protocols, total Gn doses for ovarian stimulation, Gn days for ovarian stimulation, and endometrial thickness on trigger day showed no differences of patients among the 3 phases. However, the numbers of follicles $\geq 10 \mathrm{~mm}$ and $\geq 14 \mathrm{~mm}$ on trigger day in phase 3 were less than those numbers from either phase 1 or phase $2(P=0.001$ for number of follicles $\geq 10 \mathrm{~mm}$, and $P<$ 0.001 for number of follicles $\geq 14 \mathrm{~mm}$ ). 
Table 2

Comparisons of ovarian stimulation cycle related characteristics

\begin{tabular}{|c|c|c|c|c|c|}
\hline & All & & Phase 2 & & $\begin{array}{l}P \\
\text { value }\end{array}$ \\
\hline & $N=200$ & $N=49$ & $N=81$ & $N=70$ & \\
\hline Current cycle number & & & & & 0.934 \\
\hline 1 st cycle & $144 / 72.00 \%$ & $34 / 69.39 \%$ & $59 / 72.84 \%$ & $51 / 72.86 \%$ & \\
\hline 2nd cycle & $45 / 22.50 \%$ & $13 / 26.53 \%$ & $17 / 20.99 \%$ & $15 / 21.43 \%$ & \\
\hline$\geq 3$ cycles & $11 / 5.50 \%$ & $2 / 4.08 \%$ & $5 / 6.17 \%$ & $4 / 5.71 \%$ & \\
\hline Stimulation protocol & & & & & 0.760 \\
\hline GnRH agonist & $121 / 60.50 \%$ & $28 / 57.14 \%$ & $51 / 62.96 \%$ & $42 / 60.00 \%$ & \\
\hline GnRH antagonist & $70 / 35.00 \%$ & $20 / 40.82 \%$ & $28 / 34.57 \%$ & $22 / 31.43 \%$ & \\
\hline Others & $9 / 4.50 \%$ & $1 / 2.04 \%$ & $2 / 2.47 \%$ & $6 / 8.57 \%$ & \\
\hline Gn dose (IU) & $\begin{array}{l}2162.5(1500, \\
2775)\end{array}$ & $\begin{array}{l}1875(1388 \\
2550)\end{array}$ & $\begin{array}{l}2225(1650 \\
2850)\end{array}$ & $\begin{array}{l}2288(1575 \\
2888)\end{array}$ & 0.117 \\
\hline Gn duration (days) & $11(9,12.5)$ & $10(8,12)$ & $11(9,13)$ & $11(8.75,13)$ & 0.407 \\
\hline $\begin{array}{l}\text { Number of follicles } \geq 10 \mathrm{~mm} \text { on } \\
\text { trigger day }\end{array}$ & $11.18 \pm 4.79$ & $12.00 \pm 4.27^{\#}$ & $12.28 \pm 5.14^{\star}$ & $9.33 \pm 4.17$ & $<0.001$ \\
\hline $\begin{array}{l}\text { Number of follicles } \geq 14 \mathrm{~mm} \text { on } \\
\text { trigger day }\end{array}$ & $8.21 \pm 3.31$ & $8.76 \pm 2.97^{\#}$ & $8.88 \pm 3.46^{\#}$ & $7.04 \pm 3.09$ & 0.001 \\
\hline $\begin{array}{l}\text { Endometrial thickness on } \\
\text { trigger day }(\mathrm{mm})\end{array}$ & $10.14 \pm 2.18$ & $10.45 \pm 2.17$ & $10.26 \pm 2.20$ & $10.05 \pm 2.69$ & 0.660 \\
\hline Fertilization method & & & & & 0.058 \\
\hline IVF & $149 / 74.50 \%$ & $41 / 83.67 \%$ & $63 / 77.78 \%$ & $45 / 64.29 \%$ & \\
\hline ICSI & $43 / 21.50 \%$ & $7 / 14.29 \%$ & $14 / 17.28 \%$ & $22 / 31.43 \%$ & \\
\hline Half-ICSI & $6 / 3.00 \%$ & $1 / 2.04 \%$ & $3 / 3.70 \%$ & $2 / 2.86 \%$ & \\
\hline Abbreviations: Gn, gonadotropin; & F, in vitro fertili & ion; ICSI, intra-C & toplasmic spern & injection. & \\
\hline \# denotes a significant difference & vhen comparing & tients betweer & hase 1 and ph & & \\
\hline * denotes a significant difference & hen comparing & tintc hotwe & nase 2 and $\mathrm{pl}$ & 3 & \\
\hline
\end{tabular}

Oocytes retrieval procedure related characteristics were summarized in Table 3. The operative durations were notably shortened from phase 1 to phase 2 and $3(P<0.001)$. Apparently, a significant improvement of oocyte retrival efficiencies at phase 3 was observed as compared with phase 1 and phase 2 (Table 3, Fig. 2C and 2D), regardless of the methods to determine the oocyte retrieval efficiencies. The number of retrieved oocytes, fertilization rate, number of day 3 usable embryos, number of embryos with top quality, and the vaginal bleeding rate at the puncture site showed no differences. 
Table 3

Comparisons of oocytes retrieval related features

\begin{tabular}{|c|c|c|c|c|c|}
\hline & \multirow{2}{*}{$\begin{array}{l}\text { All } \\
N=200\end{array}$} & \multirow{2}{*}{$\begin{array}{l}\text { Phase } 1 \\
N=49\end{array}$} & \multirow{2}{*}{$\begin{array}{l}\text { Phase } 2 \\
N=81\end{array}$} & \multirow{2}{*}{$\begin{array}{l}\text { Phase } 3 \\
N=70\end{array}$} & \multirow{2}{*}{$\begin{array}{l}P \\
\text { value }\end{array}$} \\
\hline & & & & & \\
\hline Operative time (min) & $10.10 \pm 4.29$ & $13.37 \pm 4.83^{\#}$ & $10.21 \pm 3.30^{*}$ & $7.67 \pm 3.24$ & $\dot{0} 001$ \\
\hline $\begin{array}{l}\text { Vaginal bleeding at } \\
\text { puncture site }(\mathrm{n} / \%)\end{array}$ & $24 / 11.00 \%$ & $6 / 12.24 \%$ & $11 / 13.58 \%$ & $5 / 7.14 \%$ & 0.429 \\
\hline Retrieved oocytes number & $8.76 \pm 3.21$ & $8.51 \pm 2.95$ & $9.07 \pm 3.27$ & $8.56 \pm 3.32$ & 0.511 \\
\hline $\begin{array}{l}\text { Oocytes retrieval efficiency } \\
(\%, \geq 10 \mathrm{~mm})\end{array}$ & $\begin{array}{l}78.2(61.5 \\
107.7)\end{array}$ & $\begin{array}{l}76.9(61.5 \\
92.1) \#\end{array}$ & $\begin{array}{l}70.60(60.0 \\
100.0)^{\star}\end{array}$ & $\begin{array}{l}100.0(66.7 \\
115.7)\end{array}$ & 0.002 \\
\hline $\begin{array}{l}\text { Oocytes retrieval efficiency } \\
(\%, \geq 14 \mathrm{~mm})\end{array}$ & $\begin{array}{l}104.2(86.4 \\
137.5)\end{array}$ & $\begin{array}{l}\text { 100.0 (85.70, } \\
130.3) \#\end{array}$ & $\begin{array}{l}93.30(82.3 \\
133.3)^{\star}\end{array}$ & $\begin{array}{l}121.1(100.0 \\
150.0)\end{array}$ & 0.011 \\
\hline Fertilization rate (\%) & $76.49 \pm 25.59$ & $73.24 \pm 27.22$ & $73.97 \pm 25.33$ & $83.54 \pm 20.04$ & 0.051 \\
\hline $\begin{array}{l}\text { Number of day } 3 \text { usable } \\
\text { embryos }\end{array}$ & $3(2,5)$ & $3(2,4)$ & $3.5(2,5.75)$ & $3(2,5)$ & 0.385 \\
\hline $\begin{array}{l}\text { Number of embryos with } \\
\text { top quality }\end{array}$ & $1(0,2)$ & $0(0,1.5)$ & $1(0,2)$ & $1(0,2)$ & 0.070 \\
\hline \# denotes a significant diffe & e when comp & g patients be & phase 1 and & se 3. & \\
\hline
\end{tabular}

The reproductive outcomes were demonstrated in Table 4. Part of the patients had cancelled embryo transfer. The reasons for embryo transfer cancelling has been listed in Table 4, and no differences of the cancellation rate were observed in patients among the three phases. For patients had embryos transferred, either the number or the stage of embryos showed no significant differences in patients among the three phases. The clinical pregnancy rates were similar in patients all through different phases $(45.16 \%, 62.50 \%$, and $54.00 \%$ for each phase, $P=$ $0.316)$. The ongoing pregnancy rate also did not differ notably among the three phases $(41.95 \%, 52.08 \%$, and $48.00 \%$ for phase 1,2 and 3 , individually, $P=0.680$ ) 
Table 4

Cmparisons of reproductive outcomes

\begin{tabular}{|c|c|c|c|c|c|}
\hline & All & Phase 1 & Phase 2 & Phase 3 & $P$ value \\
\hline & $N=200$ & $N=49$ & $\mathrm{~N}=\mathbf{8 1}$ & $N=70$ & \\
\hline $\begin{array}{l}\text { Cycles with } \\
\text { cancelled } \\
\text { embryo } \\
\text { transferred }\end{array}$ & $71 / 35.50 \%$ & $18 / 36.73 \%$ & $33 / 40.74 \%$ & $20 / 28 . .57 \%$ & 0.110 \\
\hline $\begin{array}{l}\text { Risk of } \\
\text { OHSS }\end{array}$ & $7 / 9.86 \%$ & $1 / 5.56 \%$ & $5 / 15.15 \%$ & $1 / 5.00 \%$ & \\
\hline $\begin{array}{l}\text { Elevated } \\
\text { progesterone }\end{array}$ & $7 / 9.86 \%$ & $2 / 11.11 \%$ & $3 / 9.09 \%$ & $2 / 10.00 \%$ & \\
\hline $\begin{array}{l}\text { Endometrial } \\
\text { disorders }\end{array}$ & $12 / 16.90 \%$ & $2 / 11.11 \%$ & $5 / 15.15 \%$ & $5 / 25.00 \%$ & \\
\hline Hydrosalpinx & $1115.49 \%$ & $2 / 11.11 \%$ & 6/18.18\% & $3 / 15.00 \%$ & \\
\hline $\begin{array}{l}\text { No usable } \\
\text { embryos }\end{array}$ & $24 / 33.80 \%$ & $9 / 50.00 \%$ & $9 / 27.27 \%$ & $6 / 30.00 \%$ & \\
\hline PGT & $4 / 5.63 \%$ & $1 / 5.56 \%$ & $26.06 \%$ & $1 / 5.00 \%$ & \\
\hline $\begin{array}{l}\text { Personal } \\
\text { reasons }\end{array}$ & $6 / 8.45 \%$ & $1 / 5.56 \%$ & $3 / 9.09 \%$ & $2 / 10.00 \%$ & \\
\hline $\begin{array}{l}\text { Cycles with } \\
\text { embryo } \\
\text { transferred }\end{array}$ & $129 / 64.50 \%$ & $31 / 63.27 \%$ & $48 / 59.26 \%$ & $50 / 71.43 \%$ & \\
\hline One embryo & $84 / 65.12 \%$ & $2270.97 \%$ & $34 / 70.83 \%$ & $28 / 56.00 \%$ & 0.990 \\
\hline $\begin{array}{l}\text { Two } \\
\text { embryos }\end{array}$ & $45 / 34.88 \%$ & $9 / 29.03 \%$ & $14 / 29.17 \%$ & $22 / 44.00 \%$ & \\
\hline Cleavege & $103 / 79.84 \%$ & $26 / 83.87 \%$ & $38 / 79.17 \%$ & $39 / 78.00 \%$ & 0.605 \\
\hline Blastocyst & $26 / 20.16 \%$ & $5 / 16.13 \%$ & $10 / 20.83 \%$ & $11 / 22.00 \%$ & \\
\hline \multicolumn{6}{|l|}{$\begin{array}{l}\text { Reproductive } \\
\text { outcomes }\end{array}$} \\
\hline $\begin{array}{l}\text { Biochemical } \\
\text { pregnancy } \\
(\mathrm{n} / \%)\end{array}$ & $2 / 1.55 \%$ & $0 / 0 \%$ & $0 / 0 \%$ & $2 / 4 \%$ & 0.203 \\
\hline $\begin{array}{l}\text { Clinical } \\
\text { pregnancy } \\
(\mathrm{n} / \%)\end{array}$ & $71 / 55.04 \%$ & $14 / 45.16 \%$ & $30 / 62.50 \%$ & $27 / 54.00 \%$ & 0.316 \\
\hline $\begin{array}{l}\text { Miscarriage } \\
(\mathrm{n} / \%)\end{array}$ & 8/11.27\% & $0 / 0 \%$ & $5 / 16.67 \%$ & $3 / 11.11 \%$ & 0.270 \\
\hline $\begin{array}{l}\text { Ectopic } \\
\text { pregnancy } \\
(\mathrm{n} / \%)\end{array}$ & $1 / 0.78 \%$ & $1 / 3.23 \%$ & $0 / 0 \%$ & $0 / 0 \%$ & 0.313 \\
\hline
\end{tabular}

Abbreviation: OHSS, ovarian hyper-stimulation syndrome. PGT, pre-implantation genetic test. 


\begin{tabular}{|c|c|c|c|c|c|}
\hline & All & Phase 1 & Phase 2 & Phase 3 & $P$ value \\
\hline & $N=200$ & $N=49$ & $N=81$ & $N=70$ & \\
\hline $\begin{array}{l}\text { Ongoing } \\
\text { pregnancy } \\
(\mathrm{n} / \%)\end{array}$ & $62 / 48.06 \%$ & $13 / 41.95 \%$ & $25 / 52.08 \%$ & $24 / 48.00 \%$ & 0.680 \\
\hline
\end{tabular}

Abbreviation: OHSS, ovarian hyper-stimulation syndrome. PGT, pre-implantation genetic test.

The multilinear regressions (Table 5) were performed to identify the predictor(s) of oocytes retrieval efficiencies. As demonstrated in Table 5, after adjusting several confounding factors, the phases of learning curve are the only independent predictor of oocytes retrieval rate. 
Table 5

Multiple linear regression coefficients for oocytes retrieval efficiencies

\begin{tabular}{|c|c|c|c|c|c|c|}
\hline \multirow[t]{2}{*}{ Outcomes } & \multicolumn{3}{|c|}{ Univariate analysis } & \multicolumn{3}{|c|}{ Multivariate analysis } \\
\hline & Coefficiency & $95 \% \mathrm{Cl}$ & $P$ & Coefficiency & $95 \% \mathrm{Cl}$ & $P$ \\
\hline \multicolumn{7}{|c|}{ Oocytes retrieval efficiency } \\
\hline \multicolumn{7}{|c|}{$\begin{array}{l}(\%, \text { out of follicles sized } \geq \\
10 \mathrm{~mm})\end{array}$} \\
\hline \multicolumn{7}{|l|}{ Learning phases } \\
\hline Phase 1 & -32.255 & $\begin{array}{l}-53.365 \\
-11.145\end{array}$ & 0.003 & -22.602 & $\begin{array}{l}-46.991 \\
1.787\end{array}$ & 0.069 \\
\hline Phase 2 & -26.006 & $\begin{array}{l}-44.501 \\
-7.510\end{array}$ & 0.006 & -21.704 & $\begin{array}{l}-40.938 \\
-2.470\end{array}$ & 0.027 \\
\hline Phase 3 & Ref & & & Ref & & \\
\hline Age (years old) & 0.498 & $-1.255,2.251$ & 0.576 & & & \\
\hline AFC & -0.822 & $-1.933,0.288$ & 0.146 & & & \\
\hline $\mathrm{AMH}(\mathrm{ng} / \mathrm{ml})$ & -1.679 & $-4.901,1.543$ & 0.305 & & & \\
\hline Gn days (days) & -0.176 & $-3.536,3.184$ & 0.918 & & & \\
\hline Gn doses (IU) & 0.002 & $-0.006,0.010$ & 0.638 & & & \\
\hline Endometrium (mm) & -0.634 & $-4.112,2.844$ & 0.720 & & & \\
\hline Operative time (min) & -2.741 & $\begin{array}{l}-4.626 \\
-0.857\end{array}$ & 0.005 & -1.695 & $\begin{array}{l}-3.861 \\
0.472\end{array}$ & 0.124 \\
\hline \multicolumn{7}{|c|}{ Oocytes retrieval efficiency } \\
\hline \multicolumn{7}{|c|}{$\begin{array}{l}\text { (\%, out of follicles sized } \geq \\
14 \mathrm{~mm})\end{array}$} \\
\hline \multicolumn{7}{|l|}{ Learning phases } \\
\hline Phase 1 & -22.599 & $\begin{array}{l}-34.985 \\
-10.212\end{array}$ & $<0.001$ & -16.773 & $\begin{array}{l}-31.078 \\
-2.468\end{array}$ & 0.022 \\
\hline Phase 2 & -20.174 & $\begin{array}{l}-31.027 \\
-9.322\end{array}$ & $<0.001$ & -17.578 & $\begin{array}{l}-28.860 \\
-6.296\end{array}$ & 0.002 \\
\hline Phase 3 & Ref & & & Ref & & \\
\hline Age (years old) & 0.100 & $-0.946,1.145$ & 0.851 & & & \\
\hline
\end{tabular}




\begin{tabular}{|c|c|c|c|c|c|c|}
\hline \multirow[t]{2}{*}{ Outcomes } & \multicolumn{3}{|c|}{ Univariate analysis } & \multicolumn{3}{|c|}{ Multivariate analysis } \\
\hline & Coefficiency & $95 \% \mathrm{Cl}$ & $P$ & Coefficiency & $95 \% \mathrm{Cl}$ & $P$ \\
\hline AFC & -0.607 & $-1.267,0.052$ & 0.071 & & & \\
\hline AMH (ng/ml) & -0.585 & $-2.508,1.339$ & 0.550 & & & \\
\hline Gn days (days) & -0.352 & $-2.354,1.649$ & 0.729 & & & \\
\hline Gn doses (IU) & 0.002 & $-0.003,0.006$ & 0.507 & & & \\
\hline Endometrium (mm) & -0.179 & $-2.252,1.894$ & 0.865 & & & \\
\hline Operative time (min) & -1.803 & $\begin{array}{l}-2.921 \\
-0.686\end{array}$ & 0.002 & -1.023 & $\begin{array}{l}-2.293 \\
0.248\end{array}$ & 0.114 \\
\hline
\end{tabular}

\section{Discussion}

At present, no standard training curriculum has been established for novice trainees in China. The training programs varied from institutions to institutions. Results from this study can shed a light on the minimal prerequired number of cases for practice before a trainee get an accreditation of performing oocytes retrieval procedure independently. A minimal number of 49 consecutive cases of oocytes retrieval were required to overcome the initial learning phase, followed with 81 cases for practice before the trainee reach competency of oocytes retrieval skills. Not only the operative time, but also the oocytes retrieval efficiency was notably improved from the initial learning phase to proficiency phase.

It is not easy to decide whether a trainee has reached proficiency of a new procedure. Learning curve based on CUSUM plot enables a quantitative analysis for the tutor about the minimal cases and training period required for the trainee to achieve stabilized skills. Operative time is the most commonly used marker of proficiency of certain skills and procedures $[2,7,8]$. It is generally acknowledged that the learning phases involved three distinctive phases [9]. During the first phase, trainees focus on the basic skills of performing the procedure and how to diminish the mistakes or complications. During the second phase, trainees can perform the procedures with acceptable skills and more confidence. And during the finale phase, trainees perform the procedures with adept skills and less cognitive pressure [9]. The learning curve based on the CUSUM of operative time in this study shows a similar trend of the learning phase. The trainee can become more proficient in the oocytes retrieval procedures as the practice case number increased. Specifically, the improvement in technique skills was demonstrated not only by operative time, but also the oocytes retrieval efficiency. In the final phase of learning curve, the trainee could safely and efficiently collect the oocytes.

There are only a few publications showing the trainee' learning performance in oocytes retrieval. Goldman et al [10] have proposed that a minimal number of 20 cases of oocyte retrievals was suitable for the trainees to achieve a proficiency level. However, the practice number is quite limited without adequate methods to detect the change of learning curve phases. Besides, the trainees perform oocytes retrieval on those with easier procedure while the experienced tutor performed the more challenging cases, which made the results of proficiency less comparable between the trainees and the tutors. Dessolle et al [11] reported that 43 cases are required before the 
performance of one trainee reached proficiency, which is similar to the case required to the acquisition phase in this study. Dessolle et al [7] reported a learning curve from CUSUM data of oocyte retrieval. However, in their study, follicles from one side of the ovary were aspirated by the trainees and the other side by the tutor using a randomized manner. Even though this method seems to be more reasonable to compare the operative performance between the trainee and the tutor, it is not quite applicable and efficient in clinical practice. Besides, their CUSUM were plotted based on the performance score (either success or failure) rather than operative time. Operative time, or the speed of operation is an useful parameter to determine CUSUM [8,12,13], including oocyte retrieval procedures [14]. In the current study, the CUSUM was determined based on the change trend of operative time, a more appropriate parameter to define the learning phases. After separation of learning phases, the operative performance can be compared among each learning phase with more details.

There is no uniform definition of oocyte retrieval efficiency or oocyte retrieval rate. In most reproductive institutions, follicles with diameter $\geq 10 \mathrm{~mm}$ were aspirated during oocyte collection procedures. Thus the oocyte retrieval rate was usually determined as the ratio of the number of oocytes collected to the number of follicles $\geq$ $10 \mathrm{~mm}[15,16]$. However, several studies debated that reduced maturity and fertilization ability for oocytes collected from small follicles and aspiration of those follicles were not recommended $[17,18]$. One study suggested that oocyte retrieval efficiency should be based on expected number of oocytes collected from follicles sized $\geq 14 \mathrm{~mm}$ [11]. Due to the discordant definition, both methods to determine the oocyte retrieval efficiencies were evaluated in the present study. As expected, using either method to determine oocyte retrieval efficiency, a similar trend of fluctuation in oocyte retrieval efficiency was observed.

One of the key points during oocytes retrieval is to stabilized probe and correct orientation in the vaginal [19]. We believe that with further practice, especially after the operative time reaches a plateau, the trainee got more confidence, less anxiety, and better knowledge on maintaining a stable probe. Thus, unsurprisingly, after the further training during the acquisition phase, the operative time shortened further with improvement in oocytes retrieval rate during proficiency phase. The number of retrieved oocytes, fertilization rate, and reproductive outcomes were stable across the three consecutive phases, which suggest that the trainee gradually acquire the oocytes retrieval skills without compromising patients' reproductive outcomes. After eliminating the confounding variables which might affect the oocyte retrieval rate, phases of learning curve seem to be the only variable that influence the oocytes retrieval. Data here further implies that an oocyte retrieval procedure is operator-dependent and the techniques can be improved with cumulative practice. And it is also support the necessity of establishment of technique training schedule.

The operative time and difficulties of techniques varies significantly among patients with different ovarian stimulation responses, and also locations of the ovaries. During the training program, almost only those with normal ovarian stimulation response and good prognosis were assigned for the novice trainee. Interestingly, we did observe that patients from phase 3 showed reduced ovarian reserve as depicted by lower AMH, AFC and numbers of follicles $\geq 10$ and $14 \mathrm{~mm}$ on trigger day. This phenomenon was probably because trainees were assigned with patients with good prognosis in the early phases of learning program, whereas more complex or sub-optimal prognosis patients in the later phases of learning. However, due to the improvement in oocytes retrieval rate, the retrieved number of oocytes did not show a reduced trend compared to those in phase 1 and phase 2. 
There were no severe complications occurred in this study. The most common and minor complication in oocytes retrieval was the vaginal bleeding at puncture site, which could be easily controlled by local compression. The vaginal bleeding at puncture site happened evenly throughout the 3 phases of learning period, which indicate that the safety issues might not be related with trainees' experience. Given the stable number of oocytes retrieved, stable reproductive outcomes, and no severe complications during the learning period, a trainee might be underperformed in the beginning of learning curve, the trainee was still a responsible operator, without sacrificing the reproductive outcome and safety of the patients.

To the best of our knowledge, there are very limited reports on the learning curve of transvaginal ultrasound guided oocytes retrieval, and this study provided a largest sample size and quantitative parameters for analysis of learning curve. However, there are several limitations that we should acknowledged. First, even though the study was conducted at the locally largest and high-volume center with more than 9000 oocyte retrieval cycles per year, we cannot ignore the drawback that the study was conduct at one single center retrospectively. Another limitation of the study is that all procedures were conducted by a single operator. The length of learning process could varied largely among trainees [20]. Hence, the results of this study are not applicable to every individual with various backgrounds and training experiences. An individual tailored learning program can be established based on the individual CUSUM curve. Lastly, the stress levels of the trainee across the phases of learning curve were not recorded and evaluated, which might potentially influence their technical skills.

\section{Conclusion}

In conclusion, trainees' experience in transvaginal ultrasound guided oocytes retrieval can be expected to acquire a stabilized procedure skills after 49 consecutive cases, and reaching about 10-minture operative time. Continued improvements in operative time and oocytes retrieval rate were noted with additional cases and experience. Here from our results of learning curve, we provide some implications on how to optimize the training curricula of oocytes retrieval procedure. With the help of these tips and caveats from learning curve, the establishment of training program should be more feasible and effective.

\section{Declarations}

\section{Ethics approval and consent to participate}

The study protocol was approved by the local ethics committee (Approval No. 2020-037) of The Third Affiliated Hospital of Guangzhou Medical University (see Supplemental materials for the scanned ethics approval).

\section{Consent for publication}

Each participant had signed the consent form for the use of her personal data for research use and publication.

\section{Availability of data and materials}

The datasets used and/or analysed during the current study are available from the corresponding author on reasonable request.

\section{Competing interests}

All authors have declared no conflict of interest.

\section{Funding}


This work was supported by the National Natural Science Foundation of China (Grant No. 81701400, this funding body was used for data collection, data analysis, and manuscript writing), Natural Science Foundation of Guangdong Province (Grant No. 2017A030310447, this funding body was used for data collection, data analysis, and manuscript writing), and Special Funding Program for Clinical Research from Chinese Medical Association (Grant No. 16020340650, this funding body was used for study design).

\section{Authors' contributions}

M.C. analyzed and interpreted the patients' data, and was a major contributor in writing the manuscript. D.Y. analyzed the data and contributed in manuscript drafting. S.L. collected and interpreted the patients' data. Y.W. collected and analyzed the patients' data. H.L. collected the data. Q.H. collected the data. J.L. designed the study and collected the data. All authors read and approved the final manuscript.

\section{Acknowledgement}

Not applicable.

\section{Authors' information}

\# Corresponding author: Jianqiao Liu, Department of Reproductive Medicine, The Third Affiliated Hospital of Guangzhou Medical University, 63 Duobao Road, Guangzhou, Guangdong, post code, 510000, China. (E-mail: ljq88gz@163.com, phone: +86-20-81292233.)

\section{References}

1. Bai F, Wang DY, Fan YJ, et al. Assisted reproductive technology service availability, efficacy and safety in mainland China: 2016. Hum Reprod. 2020;35(2):446-52. doi:10.1093/humrep/dez245.

2. Holzhey DM, Seeburger J, Misfeld M, et al. Learning minimally invasive mitral valve surgery: a cumulative sum sequential probability analysis of 3895 operations from a single high-volume center. Circulation. 2013;128(5):483-91. doi:10.1161/circulationaha.112.001402.

3. Ferraretti AP, La Marca A, Fauser BC, et al. ESHRE consensus on the definition of 'poor response' to ovarian stimulation for in vitro fertilization: the Bologna criteria. Hum Reprod. 2011;26(7):1616-24. doi:10.1093/humrep/der092.

4. Roque M, Haahr T, Geber S, et al. Fresh versus elective frozen embryo transfer in IVF/ICSI cycles: a systematic review and meta-analysis of reproductive outcomes. Hum Reprod Update. 2019;25(1):2-14. doi:10.1093/humupd/dmy033.

5. Jahromi BN, Mosallanezhad Z, Matloob N, et al. The potential role of granulosa cells in the maturation rate of immature human oocytes and embryo development: A co-culture study. Clin Exp Reprod Med. 2015;42(3):111-7. doi:10.5653/cerm.2015.42.3.111.

6. Van Den Abbeel E, Balaban B, Ziebe S, et al. Association between blastocyst morphology and outcome of single-blastocyst transfer. \#N/A. 2013;27(4):353-61. doi:10.1016/j.rbmo.2013.07.006.

7. Dessolle L, Biau DJ, De Larouziere V, et al. Learning curve of vitrification assessed by cumulative summation test for learning curve (LC-CUSUM). Fertil Steril. 2009;92(3):943-5. doi:10.1016/j.fertnstert.2009.01.133. 
8. Pernar LIM, Robertson FC, Tavakkoli A, et al. An appraisal of the learning curve in robotic general surgery. Surg Endosc. 2017;31(11):4583-96. doi:10.1007/s00464-017-5520-2.

9. Forbes N, Mohamed R, Raman M. Learning curve for endoscopy training: Is it all about numbers? Best Pract Res Clin Gastroenterol. 2016;30(3):349-56. doi:10.1016/j.bpg.2016.04.003.

10. Goldman KN, Moon KS, Yauger BJ, et al. Proficiency in oocyte retrieval: how many procedures are necessary for training? Fertil Steril. 2011;95(7):2279-82. doi:10.1016/j.fertnstert.2011.02.055.

11. Dessolle L, Leperlier F, Biau DJ, et al. Proficiency in oocyte retrieval assessed by the learning curve cumulative summation test. \#N/A. 2014;29(2):187-92. doi:10.1016/j.rbmo.2014.03.016.

12. Vieira A, Bourdages-Pageau E, Kennedy K, et al. The learning curve on uniportal video-assisted thoracic surgery: An analysis of proficiency. J Thorac Cardiovasc Surg. 2019. doi:10.1016/j.jtcvs.2019.11.006.

13. Kayani B, Konan S, Huq SS, et al. Robotic-arm assisted total knee arthroplasty has a learning curve of seven cases for integration into the surgical workflow but no learning curve effect for accuracy of implant positioning. Knee Surg Sports Traumatol Arthrosc. 2019;27(4):1132-41. doi:10.1007/s00167-018-5138-5.

14. Soave I, D'angelo A, Piva I, et al. A Pilot Study on Oocyte Retrieval Simulator: A New Tool for Training? J Med Syst. 2019;43(7):202. doi:10.1007/s10916-019-1340-3.

15. Lu X, Hong Q, Sun L, et al. Dual trigger for final oocyte maturation improves the oocyte retrieval rate of suboptimal responders to gonadotropin-releasing hormone agonist. Fertil Steril. 2016;106(6):1356-62. doi:10.1016/j.fertnstert.2016.07.1068.

16. Benaglia L, Busnelli A, Biancardi R, et al. Oocyte retrieval difficulties in women with ovarian endometriomas. \#N/A. 2018;37(1):77-84. doi:10.1016/j.rbmo.2018.03.020.

17. Bergh $\mathrm{C}$, Broden $\mathrm{H}$, Lundin $\mathrm{K}$, et al. Comparison of fertilization, cleavage and pregnancy rates of oocytes from large and small follicles. Hum Reprod. 1998;13(7):1912-5. doi:10.1093/humrep/13.7.1912.

18. Triwitayakorn A, Suwajanakorn S, Pruksananonda K, et al. Correlation between human follicular diameter and oocyte outcomes in an ICSI program. J Assist Reprod Genet. 2003;20(4):143-7. doi:10.1023/a:1022977002954.

19. D'angelo A, Panayotidis C, Amso N, et al. Recommendations for good practice in ultrasound: oocyte pick up(dagger). Hum Reprod Open. 2019;2019(4):hoz025. doi:10.1093/hropen/hoz025.

20. Dessolle L, Freour T, Barriere P, et al. How soon can I be proficient in embryo transfer? Lessons from the cumulative summation test for learning curve (LC-CUSUM). Hum Reprod. 2010;25(2):380-6. doi:10.1093/humrep/dep391.

\section{Figures}



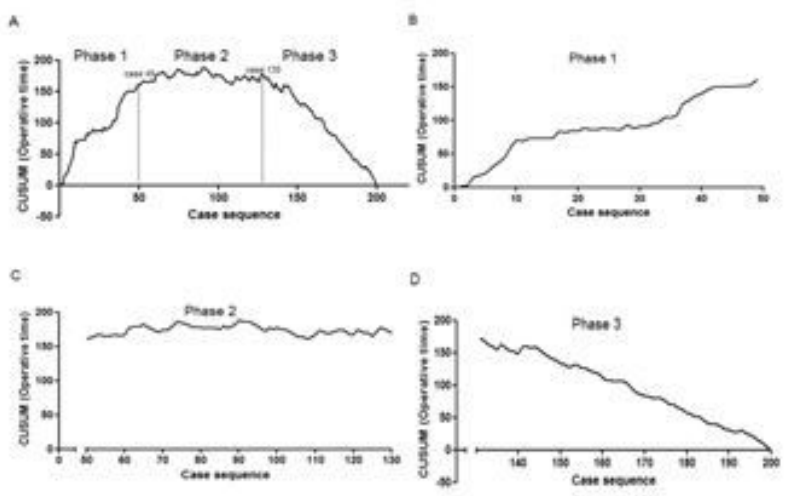

\section{Figure 1}

Learning curve (3-phase) based on CUSUM plot.
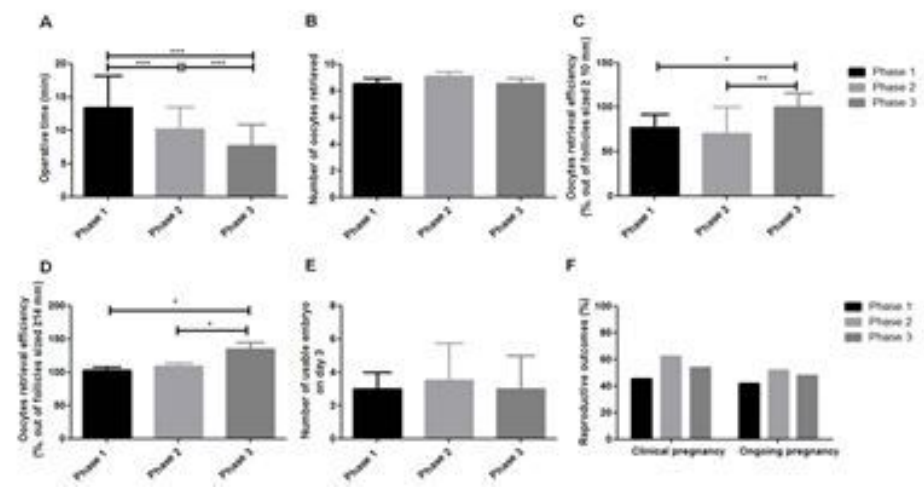

\section{Figure 2}

Comparisons of critical variables of oocytes retrieval procedure. 